Research Article

\title{
Construction Cost Simulation Based on Artificial Intelligence and BIM
}

\author{
Xingliang Du \\ Henan Finance University, Zhengzhou, Henan 451464, China \\ Correspondence should be addressed to Xingliang Du; duxingliang@hafu.edu.cn
}

Received 25 October 2021; Accepted 8 November 2021; Published 28 November 2021

Academic Editor: Le Sun

Copyright (c) 2021 Xingliang Du. This is an open access article distributed under the Creative Commons Attribution License, which permits unrestricted use, distribution, and reproduction in any medium, provided the original work is properly cited.

\begin{abstract}
Quickly estimating the main engineering quantity and project cost of the project is conducive to the management staff to have an overall grasp of the project in the early stage and to grasp the development direction of the project in advance. Moreover, it can play an important guiding role in the further construction management of the project and can help managers prevent the emergence of unfavorable factors in the project, make corresponding construction deployments, and avoid risks. This paper combines artificial intelligence technology to construct a construction project cost simulation system. In this system, BIM is mainly used to simulate construction engineering, and the construction engineering cost is simulated and analyzed in combination with the pricing file. Finally, the results of experimental research show that the intelligent model proposed in this paper can play an important role in the cost of construction projects.
\end{abstract}

\section{Introduction}

Engineering cost management has a long history of development. Modern engineering cost management theory originated in the United Kingdom. Since then, various countries and regions have combined their own characteristics to form different project cost management models. Based on different management models, it is mainly divided into several models represented by the United Kingdom, the United States, Japan, Singapore, Malaysia, and Hong Kong. China's modern engineering cost management theory started late, but its development is rapid. After the founding of the People's Republic of China, China established a set of quota management systems compatible with the planned economy by referring to the former Soviet Union's engineering construction management model, which played a very important role in the recovery and development of the national economy. After the reform and opening up, marketization has continued to deepen, and the original management model can no longer meet the requirements of the modern and rapid development of the market, and China's engineering cost industry has also ushered in a golden period of development. The basis and methods of project pricing have been continuously reformed, the project cost management system has been continuously improved, and the project cost consulting industry has developed rapidly. Moreover, China's engineering cost system has also changed from the original fixed-rate pricing model to the list-based pricing model. Now, China's project cost management is increasingly showing the development trend of internationalization, informatization, and specialization.

Although the traditional method of quota or list measurement and pricing is accurate, it also has the characteristics of long working hours, large manpower input, large workload, and high requirements for the accuracy of drawings. In today's construction market, bidders are required to quickly give quotations, and time requirements are high. Bidding companies often can only make bid quotations based on personal experience in the past. This offer relies heavily on personal engineering experience and lacks credibility. The rapid estimation of main engineering quantities and engineering costs fills in the shortcomings of traditional quota or list measurement and pricing methods that are time-consuming and large in workload and can meet the high-efficiency requirements in the modern market. At the same time, the model foundation of this method is based 
on the data samples of multiple completed projects in the market, and the credibility of the indicators is high, and it provides important data and theoretical support for the decision-making of bidding and quotation [1].

The control of project cost is an important factor for the project to achieve better economic benefits. Extensive management and cost control will firstly lead to unnecessary waste of social resources and secondly make the project always in a state of unknown results, which is not good for the long-term development of the enterprise [2]. During the project, the management personnel can quickly understand the actual development of the project and analyze the degree of deviation of the project progress by comparing the corresponding project cost indicators of the actual completed project. At the same time, they can followup the problems in the project in time and adjust the project plan [3].

Based on this, this paper explores the construction of a construction project cost simulation system combined with artificial intelligence technology. In this system, BIM is mainly used to simulate the construction project to improve the accuracy of the construction project cost.

\section{Related Work}

In recent years, with the impact of foreign construction markets and the promotion of national policies, many large enterprises in the domestic industry have been actively exploring the use of BIM in order to improve their international competitiveness. Some construction projects will require $\mathrm{BIM}$ to be written into the bidding contract when bidding for construction projects, and BIM has gradually become a threshold for enterprises to participate in the project [4]. At present, some large and medium-sized design companies have established their own BIM teams and continue to accumulate practical experience. Although construction companies started late, they have been advancing in exploration and have achieved certain results [5]. As companies from all walks of life attach importance to $\mathrm{BIM}$, the demand for BIM talents also arises. BIM technology not only brings about the advancement and upgrading of existing technologies and realizes the leapforward development of the construction industry but also indirectly affects the production organization mode and management method and will affect people's way of thinking in the longer term [6].

The main work in the decision-making stage is to compare plans and conduct feasibility studies on the project. Through the use of BIM technology to compare the cost of multiple plans and other aspects [7], the most economical and reasonable investment plan can be selected. The introduction of BIM technology assists decision-makers to quickly and accurately make program choices and improve the accuracy of decision-making. If all kinds of BIM information are integrated on the cloud platform, the application of cost management in the decision-making stage is an investment estimation with the help of engineering cases in the BIM cloud platform database [8]. In the database of the BIM cloud platform, the BIM models of completed cases similar to the proposed project can be directly obtained. The staff can directly make simple modifications on the BIM model according to the investment plan and then obtain the approximate engineering quantity, cost, and other different indicators, and quickly and accurately obtain the investment estimate. This method has changed the traditional investment estimation method based on historical data and personnel work experience provided by local government departments [9]. The cloud platform database realizes reliable investment estimation of the proposed project and improves the estimation accuracy of the proposed project [10].

Literature [11] pointed out that 5D-BIM technology is a BIM technology that is based on the three spatial dimensions of BIM by adding two more dimensions, namely, schedule and cost. On the surface, 5D-BIM technology seems to incorporate two simple dimensions on the basis of BIM technology, but the significance of the integration is far beyond the added dimension itself. It is a reorganization of BIM. At the same time, the integration of the two dimensions of schedule and cost also enables BIM to be used in the management of the entire life cycle of construction projects, which can effectively improve the management level of construction projects. Literature [12] pointed out in the research process that 5D-BIM technology is actually based on BIM, which is a type of construction management technology of building information modeling. With the support of BIM, the progress, resources, budget, and construction, the organization and other key information are incorporated into the management system, and the project construction process is simulated so that the project leader and management personnel can understand the project information in a timely manner and improve the efficiency of decision-making on the project. Literature [13] pointed out in the research process that information is the core of 5D-BIM technology, and the basic elements (components or objects) of BIM as a carrier are geometric information, cost information, and related requirements and physical characteristics of project construction. Expressed in the form of parameters, through the corresponding Boolean operation and spatial topological relationship to complete the sorting of each parameter information, make it form a digital model, and finally, provide a digital model for the construction project cost management and each participating party of the project. This model can be used to communicate projectrelated work to ensure the efficiency of project management. Literature [14] focuses on the application of 5D-BIM technology in the design phase of construction projects and points out that one of the most notable features of the engineering design phase is that a large amount of information data is generated, and this type of information data is directly related to the project. Quality and management efficiency, and the application of 5D-BIM technology at this stage build an information chain of information resource sharing between various work in the project design phase so that each participating unit and the personnel of the design department can form a project. A comprehensive understanding of design information has laid a good foundation for improving the scientificity and reliability of project design. 


\section{Building Structure Simulation Based on Artificial Intelligence}

Voronoi diagram is an important geometric structure in computational geometry. The Voronoi diagram is essentially a division of the plane according to a set of discrete points on the plane. It is defined as follows:

We arbitrarily give a set $P=\left\{p_{1}, p_{2}, p_{3}, \ldots, p_{n}\right\}$ of $n$ discrete points at different positions on the plane, which is called $p_{i}(i=1,2,3, \ldots, n)$ as a station. We classify all points on the plane according to the principle of Euclidean distance and obtain the point set $V R\left(p_{i}\right)$ closest to each $p_{i}$, as shown in the following equation:

$$
V R\left(p_{i}\right)=\left\{q|| q p_{i}|\leq| q p_{j} \mid, \quad \forall j \neq i, p_{i}, p_{j} \in P\right\} .
$$

Among them, $|q p|$ represents the Euclidean distance between the two points $p$ and $q$. VR (pi) is the Voronoi area of site $p_{i}$. The Voronoi diagram VD $(P)$ of $\mathrm{P}$ is the union of the Voronoi regions of all stations, as shown in the following equation:

$$
V D(P)=U_{i=1}^{n} V R\left(p_{i}\right) .
$$

The entire plane is divided into $n$ subregions that do not cover each other, and each subregion corresponds to a site. Any point $q$ within the $p_{i}$ subarea of the site satisfies $\left|q p_{i}\right| \leq\left|q p_{j}\right|$, where $p_{j} \in P-\left\{p_{i}\right\}[15]$.

Figure 1 shows the Voronoi diagram of eight discrete points.

For a multiboundary polygon $\mathrm{P}$, if the angle $\alpha>180^{\circ}$ between the vertex pi and the two associated edges in the Voronoi diagram drawing area, then $p_{i}$ is called a concave vertex. Each concave vertex or each edge in $\mathrm{P}$ is called a site. Each site has a Voronoi region, and the union of the Voronoi regions of all sites is a Voronoi diagram of polygon $\mathrm{P}$. The Voronoi diagram of the polygon $\mathrm{P}$ is obtained in the following way:

According to different sites, there are three types of bisectors:

(1) Vertex and vertex: the bisector is the vertical bisector of the connection between the vertices, as shown in Figure 2(a)

(2) Vertex and edge: the bisector is a parabola, as shown in Figure 2(b)

(3) Side and side: the bisector is the bisector of the angle formed by the two sides, as shown in Figure 2(c)

After that, we use the above three bisectors to divide the Voronoi area of all stations. It is particularly important to note that for each concave vertex, in order to divide its Voronoi area and the Voronoi area of the two sides with it as the end point, in the area where the Voronoi diagram is drawn by the polygon, the concave vertex is the end point. Then, combine the two vertical bisectors of the edge. The Voronoi diagram of the polygon $\mathrm{P}$ is divided by the abovementioned three bisectors and the vertical bisectors of the concave vertices. Among them, the common edge between the Voronoi areas of the two sites is called the Voronoi edge,

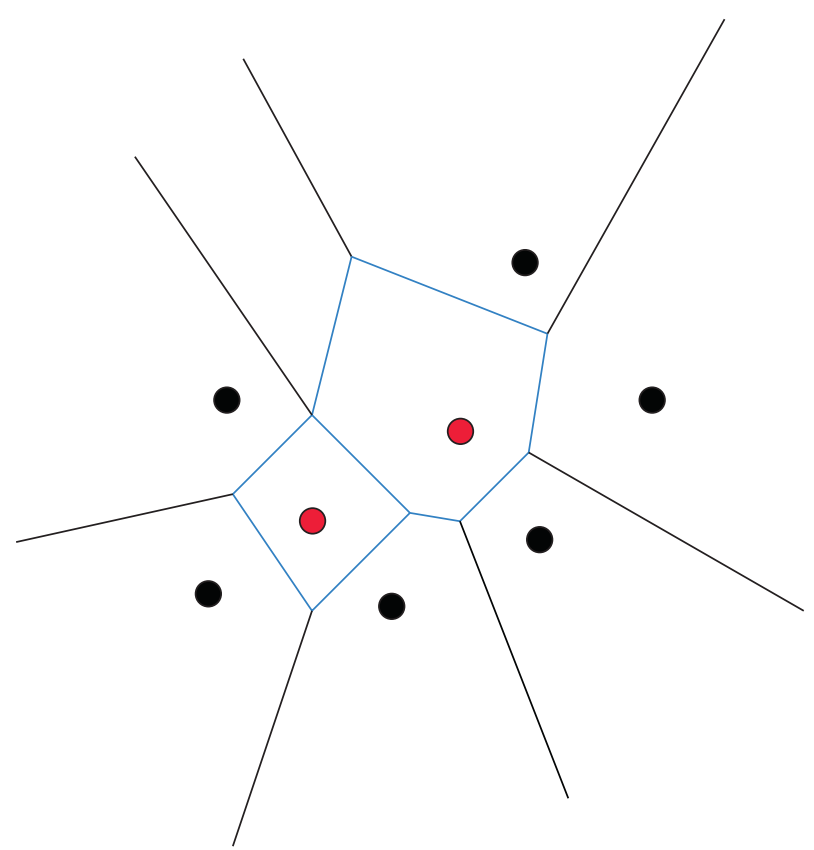

FIGURE 1: Voronoi diagram of 8 discrete points.

and the intersection of the Voronoi edges is called the Voronoi vertex. The Voronoi skeleton path of the polygon is composed of Voronoi edges and Voronoi vertices. Figure 3 shows the Voronoi diagram of a multiboundary polygon. The dotted line is the Voronoi skeleton path of the polygon.

Here, this paper introduces the concept of weak visibility. We set a polygon $P$, and $Q$ is a subset of the polygon $P$. If there is a point in $Q$ that can see a point $s$ in $P$, then $s$ is said to be weakly visible relative to $Q$. As shown in Figure 4(a), $Q$ is the edge $p_{1} p_{2}$, and the point $p_{1}$ in $Q$ can be seen at the point $s$. Therefore, the point $s$ is weakly visible with respect to $\mathrm{Q}$. That is to say, in the polygon $\mathrm{P}$, as long as the station $s$ can see a certain point in $Q$, then the point $s$ is weakly visible with respect to $Q$.

The set of all weakly visible points of $Q$ is called the weakly visible area of $Q$ in the polygon $P$, as shown in Figure 4(b).

How to quickly build an MR environment in large indoor spaces with many room structures such as museums and art galleries is discussed below. The construction method proposed in this paper includes the following 4 steps:

(1) Build the initial virtual scene

(2) Establish the mapping relationship between the virtual scene and the physical environment

(3) Set the transparency value of the wall

(4) Offset error correction

To construct the MR environment, the scene must be constructed first, and the virtual scene will be constructed according to the real environment. HoloLens completes scene construction by scanning, which is based on a multiview geometric reconstruction method. However, the scanning process is not suitable for MR applications in a 


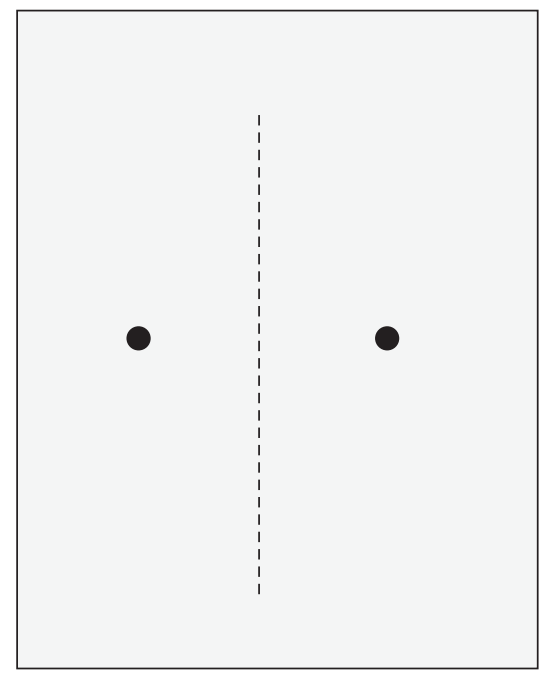

(a)

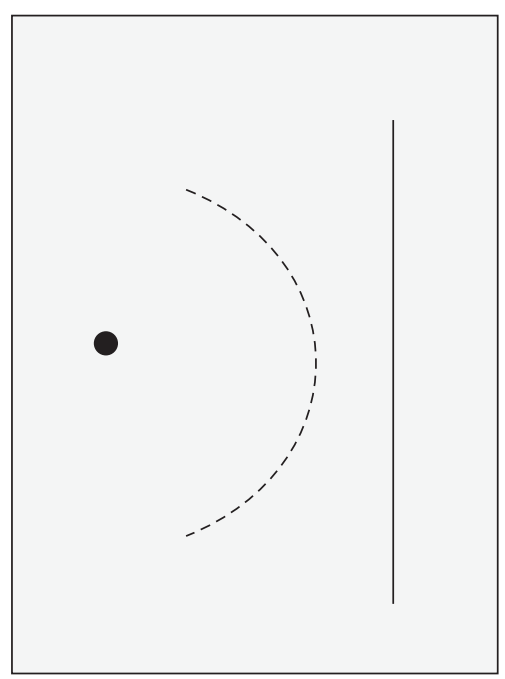

(b)

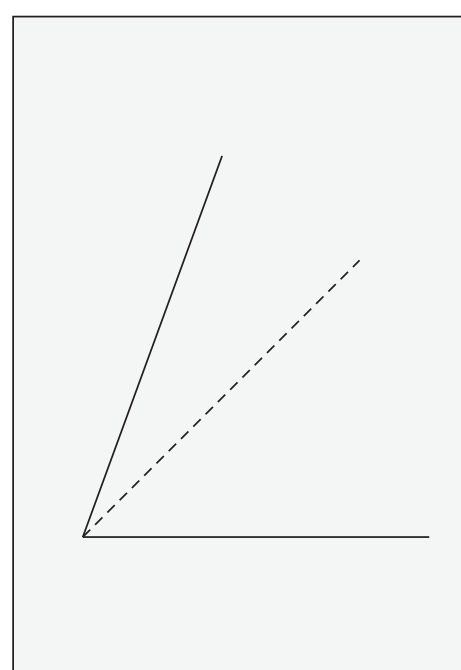

(c)

Figure 2: Three bisectors.

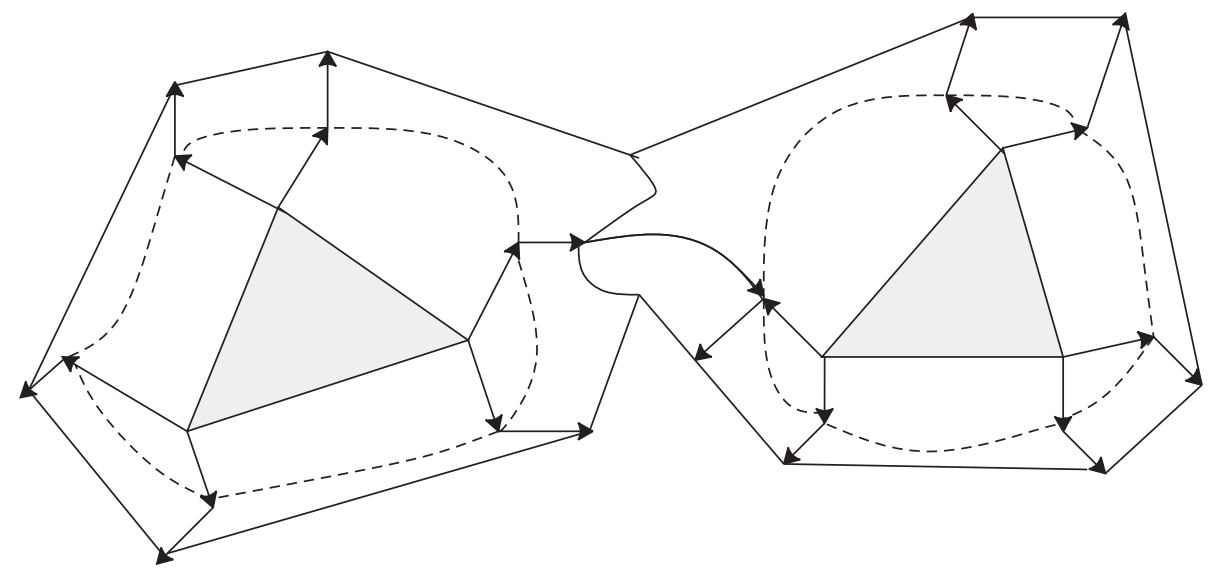

FIGURE 3: Voronai diagram of multiboundary polygon.

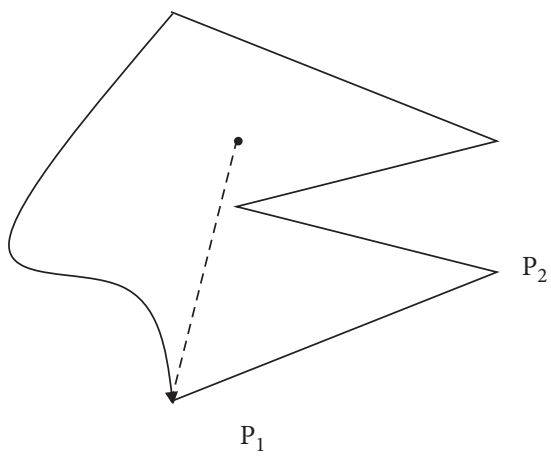

(a)

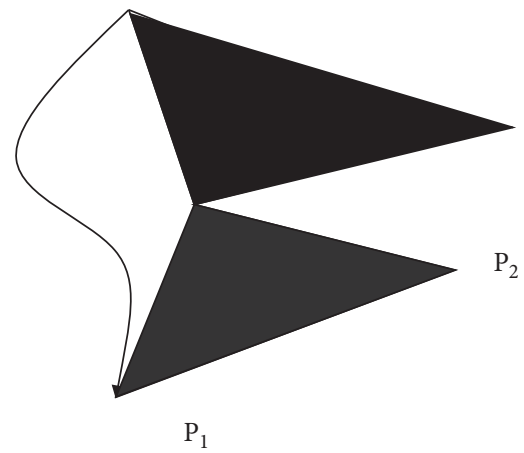

(b)

FIgURE 4: Weak visibility. (a) Weak visibility. (b) Weak visibility. 


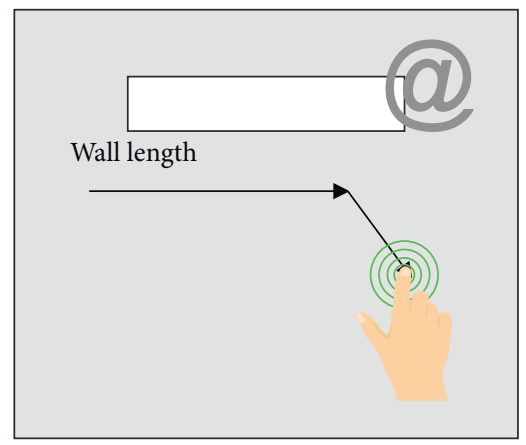

(a)

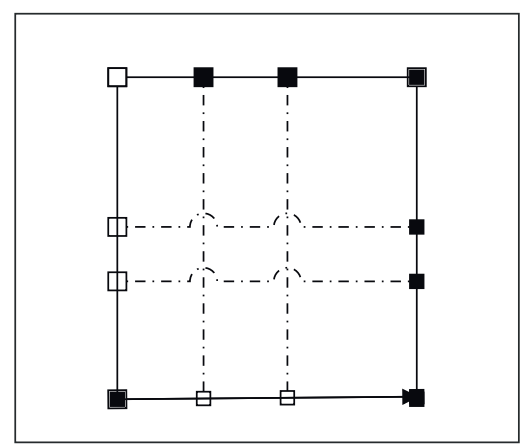

(b)

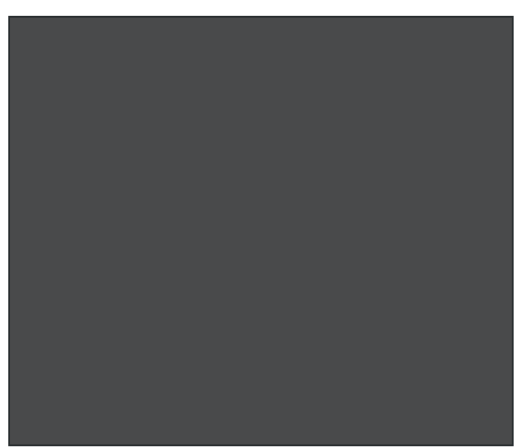

(c)

FIGURE 5: 2D structure diagram of the construction method. (a) 2D structure drawing drawn by parameters. (b) Input plane structure figure. (c) $2 \mathrm{D}$ structure figure obtained after processing.

wide range of indoor spaces. On the one hand, because the scanning process is time-consuming and laborious; on the other hand, it is impossible to scan and save the entire space at once. Therefore, this article uses the prebuilt virtual wall model to replace the cumbersome scanning process, while saving the structural information of the real environment for subsequent reuse. The specific method is as follows:

(1) This article uses three data sources to obtain a 2D wall structure diagram of the physical environment. (1) This article draws a 2D structure diagram according to the structural parameters of the real environment (as shown in Figure 5(a)), and the user can draw walls through multitouch or mouse operation. After drawing, the user can input the length of the wall in the text box to adjust the wall just drawn. (2) After inputting the plane structure diagram of the building, the $2 \mathrm{D}$ structure diagram is extracted through the edge detection algorithm (as shown in Figure 5(b)). (3) This article imports the existing spatial 3D model and extracts the $2 \mathrm{D}$ structure diagram.

(2) The algorithm imports the $2 \mathrm{D}$ structure diagram obtained in step 1 into the design end running on the surface. Then, the algorithm traverses the information of each node in the structure diagram to construct a virtual wall model.

The Figure 5(a) represents the 2D structure drawing drawn by parameters, the left figure in the Figure 5(b) is the input plane structure figure, and the right figure is the $2 \mathrm{D}$ structure figure obtained after processing.

In order to ensure the authenticity of the experience, it is necessary to establish an accurate mapping relationship between the virtual scene and the real environment, transform the virtual space and the real space into a unified coordinate system, and ensure that the virtual scene matches the real environment accurately, that is, scene registration. This article constructs a virtual space coordinate system on the design side. HoloLens can establish a world coordinate system 1:1 with the real world. This article uses this coordinate system to represent the position in the real world.

$P_{1}\left(x_{1}, z_{1}\right)$ and $P_{2}\left(x_{2}, z_{2}\right)$ are the two points in the virtual space, and $P_{1}^{\prime}\left(x_{1}^{\prime}, z_{1}^{\prime}\right)$ and $P_{2}^{\prime}\left(x_{2}^{\prime}, z_{2}^{\prime}\right)$ are the corresponding two points in the real space. First, $\overrightarrow{P_{1} P_{2}}$ and $\overrightarrow{P_{1}^{\prime} P_{2}^{\prime}}$ are calculated, respectively, as shown in the following formulas and [16]:

$$
\begin{aligned}
& \overrightarrow{P_{1} P_{2}}=(x, z)=\left(x_{2}-x_{1}, z_{2}-z_{1}\right), \\
& \overrightarrow{P_{1}^{\prime} P_{2}^{\prime}}=(x \prime, z \prime)=\left(x_{2}^{\prime}-x_{1}^{\prime}, z_{2}^{\prime}-z_{1}^{\prime}\right) .
\end{aligned}
$$

In this paper, $V(V . x, V . z)$ represents any point in virtual space, and $R(R . x, R . z)$ represents the corresponding point of $V$ in real space. According to the following formula, the corresponding point $R$ coordinate in the real world can be obtained according to the $V$ point coordinate in the virtual coordinate system, as shown in the following formula :

$$
\left\{\begin{array}{l}
R . x=\left\{\cos \theta \cdot\left(x_{1}-V \cdot x\right)-\sin \theta \cdot\left(V . z-z_{1}\right)\right\} \cdot \text { ratio }+x_{1}^{\prime}, \\
R . z=\left\{\sin \theta \cdot\left(x_{1}-V \cdot x\right)+\cos \theta \cdot\left(V . z-z_{1}\right)\right\} \cdot \text { ratio }+z_{1}^{\prime} .
\end{array}\right.
$$

Conversely, the coordinates of point $V$ can be obtained from the point $R$ according to the following formula [17]:

$$
\left\{\begin{array}{l}
V \cdot x=\left\{\cos \theta \cdot\left(x_{1}^{\prime}-R \cdot x\right)-\sin \theta \cdot\left(R \cdot y-y_{1}^{\prime}\right)\right\} \cdot\left(\frac{1}{\text { ratio }}\right)+x_{1}, \\
V \cdot y=\left\{\sin \theta \cdot\left(x_{1}^{\prime}-R \cdot x\right)+\cos \theta \cdot\left(R \cdot y-y_{1}^{\prime}\right)\right\} \cdot\left(\frac{1}{\text { ratio }}\right)+y_{1} .
\end{array}\right.
$$


Among them, the formula $\theta$ represents the angle between the vector $\overrightarrow{P_{1} P_{2}}$ and $\overrightarrow{P_{1}^{\prime} P_{2}^{\prime}}$, and the calculation method of $\sin \theta, \cos \theta$, and ratio is as shown in the following formula [18]:

$$
\begin{aligned}
& \sin \theta=(x \cdot z \prime-x \prime \cdot z) / \sqrt{x^{2}+z^{2}} \cdot \sqrt{x^{2 \prime}+{ }^{2 \prime}} \\
& \cos \theta=(x \cdot x \prime-z \prime \cdot z) / \sqrt{x^{2}+z^{2}} \cdot \sqrt{x^{2 \prime}+z^{2 \prime}} \\
& \text { ratio }=\sqrt{x^{2 \prime}+z^{2 \prime}} / \sqrt{x^{2}+z^{2}}
\end{aligned}
$$

Through the above formula, the virtual coordinate system and the real space coordinate system can be transformed into each other, and each node information in the 2D structure diagram can be traversed to find the position in the real space coordinate system, thereby constructing a virtual wall model. In addition, users can design scene content on the design side and deploy the scene content to the HoloLens experience side in real time through the above mapping relationship.

In real space, objects behind the wall cannot be seen from in front of the wall due to the occlusion of the wall. But for transparent glass and translucent frosted walls, users can see the objects behind the walls. Therefore, in order to ensure the real experience effect, this paper treats different walls differently and introduces the transparency value $\varphi$ of the virtual wall.

(1) If the walls in the physical space are opaque, this paper sets the transparency value $\varphi$ to 0 . In this case, the corresponding virtual wall generated by the above steps will be attached with a transparent material. During the roaming process, the MR application will calculate and render the virtual wall to ensure that the virtual object placed behind the real wall will be blocked. At the same time, the use of transparent materials allows the user to only see the real wall and not the virtual wall when wearing the HoloLens device. This design effectively achieves the following effects: the real wall can block the virtual objects behind the wall. As shown in Figure 6(a), the upper part shows the effect that the user looks at when there is only a real wall. The real wall is an opaque wall, and the cultural relic is a virtual cultural relic rendered by HoloLens; below is the effect that the user sees when the virtual wall exists. This paper constructs a virtual wall that is the same as a real wall, attaches a transparent material, and places it in the position of the real wall. The user cannot see the virtual wall, and the existence of the virtual wall obscures the false cultural relics.

(2) If the real wall is transparent or semitransparent, such as a wall made of glass, this paper sets the transparency value $\varphi$ of the virtual wall to a value in $(0,1)$. At this time, the virtual wall will not be calculated and rendered, and the user can see the virtual object behind the wall. But the transparency value $\varphi$ will serve as a parameter for the rendering of virtual objects. This article will introduce how to use the transparency value $g$ to accelerate the

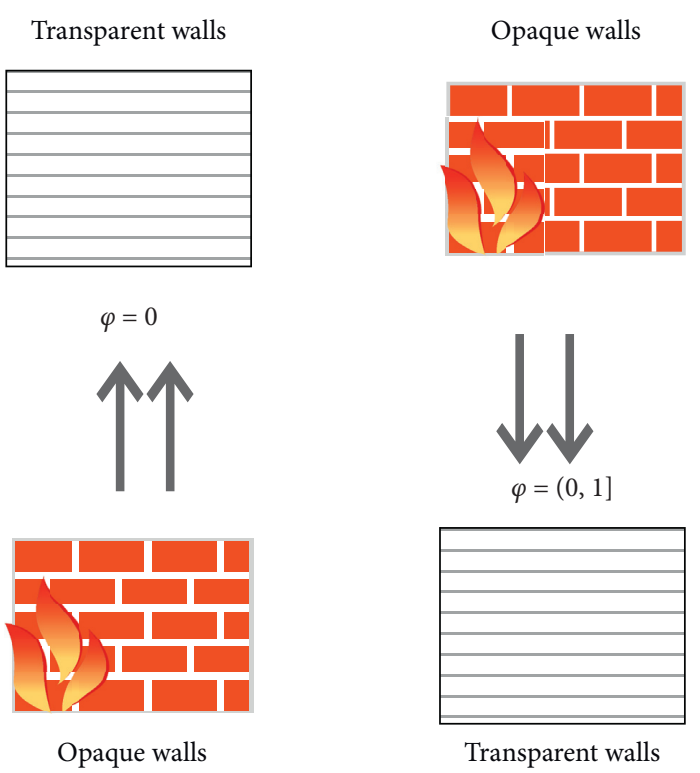

FIGURE 6: Setting the virtual wall transparency value $p$ to process different walls.

rendering of MR scenes. As shown in Figure 6(b), the upper part is the effect that the user looks at when there is only a real wall. Among them, the real wall is a translucent glass wall, and the cultural relics are virtual cultural relics rendered by HoloLens: the display effect processed with the transparency value $p$ is below. Since the glass wall is semitransparent, this paper reduces the accuracy of the virtual model and makes it rougher to speed up the rendering of the scene.

This article finds that during the user's roaming process, the virtual wall will gradually shift and cannot accurately correspond to the real wall position. In some special cases, HoloLens may not recognize its location and lose tracking. When the device recovers from this situation to a normal state, the position correspondence between the real wall and the virtual wall will also deviate. As the user's roaming time and range in the MR environment increase, the offset error will gradually accumulate and become more and more obvious, and the user's realistic experience will become worse and worse.

To solve this problem, this paper sets up some spatial anchor points in the MR environment according to the layout of the virtual scene to anchor the virtual scene in the real environment.

Based on the data structure VorPa, this paper proposes two anchor point deployment algorithms based on greedy ideas and integer linear programming methods. In the processing process, since the virtual wall needs to correspond accurately to the real wall, the wall model is regarded as a special virtual object, and the position of the virtual object is the position of the midpoint of the wall. After merging the virtual wall and the virtual object collection, all the virtual model collections that need to be covered by anchor points in the scene are obtained as $M=W \cup R=\left\{m_{1}, m_{2}, m_{3}, \ldots, m_{n}\right\}$. 
Before using the two algorithms, this paper preprocesses the scene based on the data structure VorPa:

(1) This article sets the value $v_{i}(i \in n)$ for each virtual model $m_{i}$. The value $v_{i}$ represents the visible probability of the virtual model $m_{i}$ in the scene. In other words, if the user randomly selects a position and perspective in the scene, the probability of seeing the model. The calculation method of the probability of finding the model is as follows [19]:

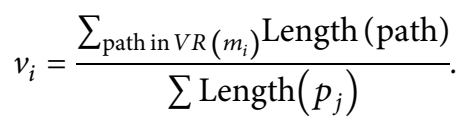

Among them, VR (mi) represents the visible area of the virtual model $m_{i}$ in the scene, and $p_{j}$ represents the $\mathrm{j}$-th roaming subpath in the scene. The calculation of $v_{i}$ is based on the length of the roaming path, that is, the ratio of the length of the roaming path that falls into the visible area of the cultural relic $m_{i}$ to the total length of the scene roaming path.

(2) This article sets the value of $v_{p_{j}}$ for each subpath. In the data structure VorPa, if the virtual model $m_{i}$ is in the weakly visible area of the path $p_{j}$, the cultural relic $m_{i}$ is bound to the model list modelList of the path pi. The sum of the value of all models in the $p_{j}$ model list modelList is the value of the path $p_{j}$, as shown in the following equation [20]:

$$
v_{p_{j}}=\sum_{m_{i} \text { in modeI List of } p_{j}} v_{i} .
$$

Next, Algorithm 1 based on greedy thought is first introduced: anchor deployment algorithm based on greedy thought.

(1) The algorithm sorts all paths according to the value of the path and selects the path $P_{\max }$ with the largest value.

(2) For the model on the path $P_{\max }$, the algorithm selects a circle with a radius of $3 \mathrm{~m}$ so that it can cover the model combination with the largest total value on the $P_{\max }$. The position of the center of the circle is the position of the anchor point $s$, which is added to the radium point set. Among them, the way to find the anchor point $s$ is as follows:

(1) The algorithm creates a two-dimensional array $\mathrm{C}$ containing all the scenes in the array $\mathrm{C}[\mathrm{a}][\mathrm{b}]$ corresponds to a point in the scene with coordinates (a, b) and assigns all the initial values of the two-dimensional array $\mathrm{C}$ to 0 . The position of the model $m_{i}$ is $\left(x_{i}, y_{i}\right)$, and the algorithm finds the intersection area between a circle with $\left(x_{i}, y_{i}\right)$ as the center and a radius of $3 \mathrm{~m}$ and the model $m_{i}$ visibility region $\operatorname{VR}\left(m_{i}\right)$. Moreover, the algorithm adds $v_{i}$ to the value of the array position corresponding to this area and traverses all models. In the model, the list of the path $P_{\max }$ does the above operation.
(2) The algorithm traverses the two-dimensional array C and finds the scene area corresponding to the array subscript with the largest value, which is the anchor point distribution area that this paper is looking for. The algorithm chooses a point as the anchor point $s$.

(3) The algorithm traverses all roaming subpaths, deletes all virtual models covered by the anchor point $s$ contained in the subpaths, and modifies the value of the path, subtracting the corresponding model value.

(4) The algorithm returns to step 1 , repeats steps 1 to 3 until the value of $P_{\max }$ is 0 , and outputs the anchor point is set.

Algorithm 1. Based on greedy thinking is not necessarily the optimal solution of the problem, and it is time-consuming and long. This article uses it as the baseline algorithm. Furthermore, this paper transforms this problem into an optimization problem, that is, minimizing the number of anchor points. After that, this paper proposes the second algorithm. Algorithm 2 is based on the idea of integer linear programming.

Algorithm 2. Anchor point deployment algorithm based the on integer linear programming is as follows:

(1) The algorithm gets the initial set of anchor points. Before constructing the mathematical model, an initial set of $l$ anchor points that can cover all virtual models in the scene is obtained through the following process:

According to the method in Algorithm 1, the algorithm creates a two-dimensional array $C$ containing all scenes and assigns all initial values to 0 . The algorithm finds the intersection area between a circle with $m_{i}\left(x_{i}, y_{i}\right)$ as the center and a radius of $3 \mathrm{~m}$ and the model $m_{i}$ visibility area $\operatorname{VR}\left(m_{i}\right)$, and the value of the array subscript position corresponding to this area is added to $v_{i}$.

The algorithm traverses the entire two-dimensional array $\mathrm{C}$, finds all anchor point positions that can cover different virtual model combinations without repeating them, and adds them to the initial anchor point set.

(2) According to the problem description, this paper constructs a mathematical model [21]:

$$
\begin{aligned}
& \min z=\sum_{i=1}^{l} s_{i}, \\
& \left\{\begin{array}{l}
s_{i}=0 \text { or } 1, i=1,2, \ldots, l, \\
\sum_{i=1}^{l} F[i][j] \cdot s_{i} \geq 1, \quad j=1,2, \ldots, n .
\end{array}\right.
\end{aligned}
$$

In this mathematical model, the variable $s_{i}$ represents the i-th anchor point. It is assumed that there are initially $l$ 


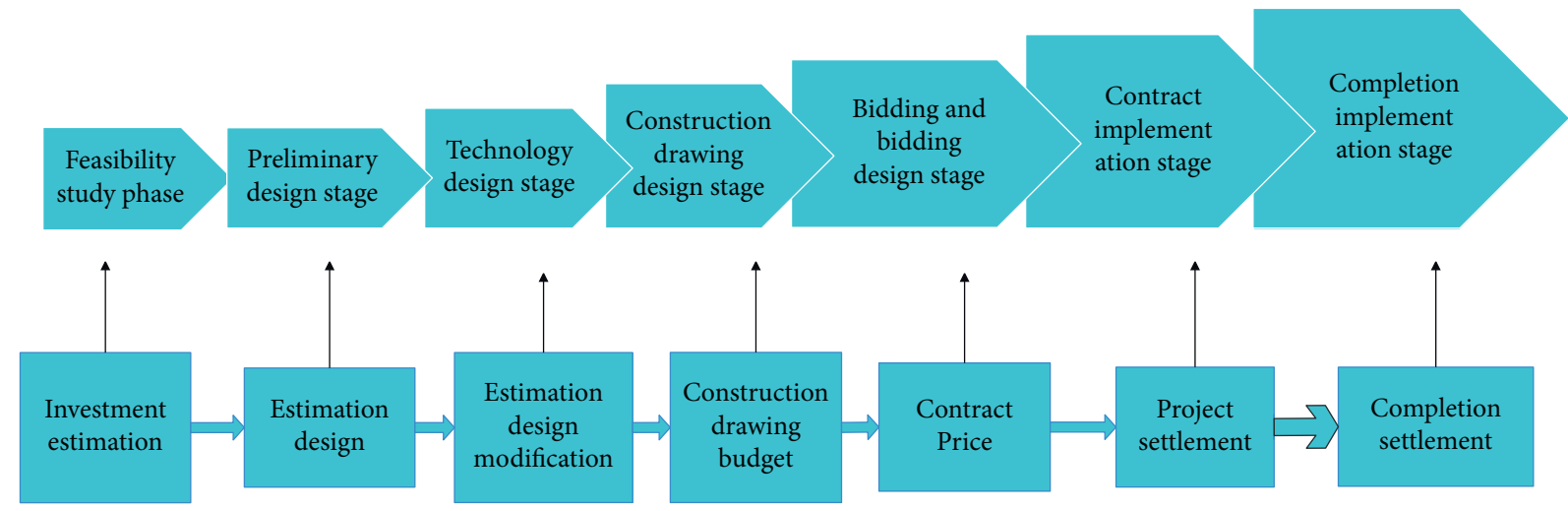

FIgURE 7: Valuation file.

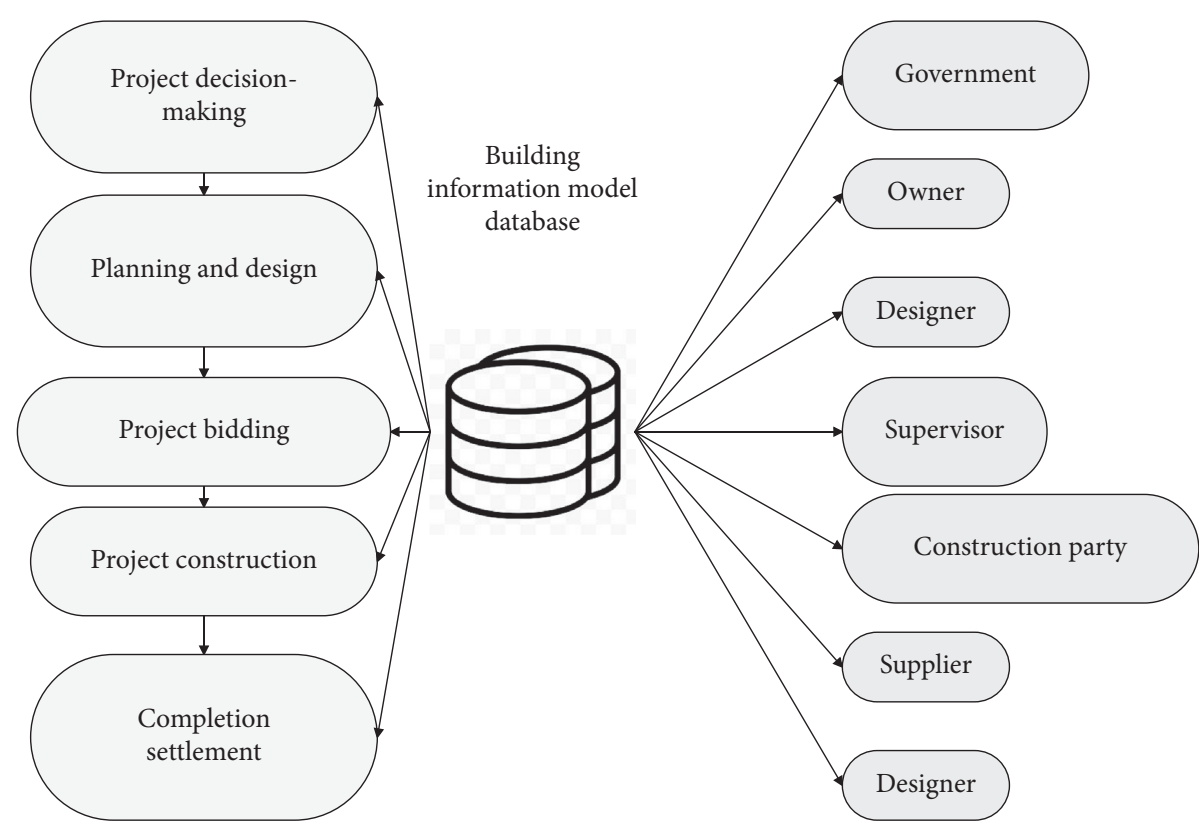

FIGURE 8: Information exchange diagram of information model.

anchor points, and these anchor points can cover all virtual models in the scene. $F[i][j]$ indicates whether the i-th anchor point can cover the $j$-th virtual model. If it can be covered, the value is 1 , and if it is not covered, the value is 0 . The goal of this paper is to minimize the number of anchor points and satisfy two constraints. (1) The value of the anchor point $s_{i}$ is 0 or 1.0 means that the $\mathrm{i}$-th anchor point is not placed, and 1 means that the $\mathrm{i}$-th anchor point is placed. (2) All models in the scene can be covered, that is, for any model, the number of anchor points that can cover the model is greater than or equal to 1 .

\section{Construction Cost Simulation Based on Artificial Intelligence and BIM}

The whole-process project cost management is the management of project cost, which is carried out in accordance with the construction procedures of the engineering project and is carried out from the feasibility study of the project-toproject decision-making, project design, bidding, project implementation to the final completion acceptance, delivery, and use, and project postevaluation. These stages all correspond to the corresponding project cost valuation documents, as shown in Figure 7.

The information exchange structure of the information model (as shown in Figure 8) and the BIM model in the refined management of the project cost (as shown in Figure 9) must be clarified before the application of BIM technology to the construction engineering calculation of the price file, which lays the foundation for further refined applications.

After constructing the above model, the model is verified and analyzed, and the BIM simulation effect of the model and the project cost evaluation effect of the model are mainly verified. The results are shown in Table 1 and Figure 10. 


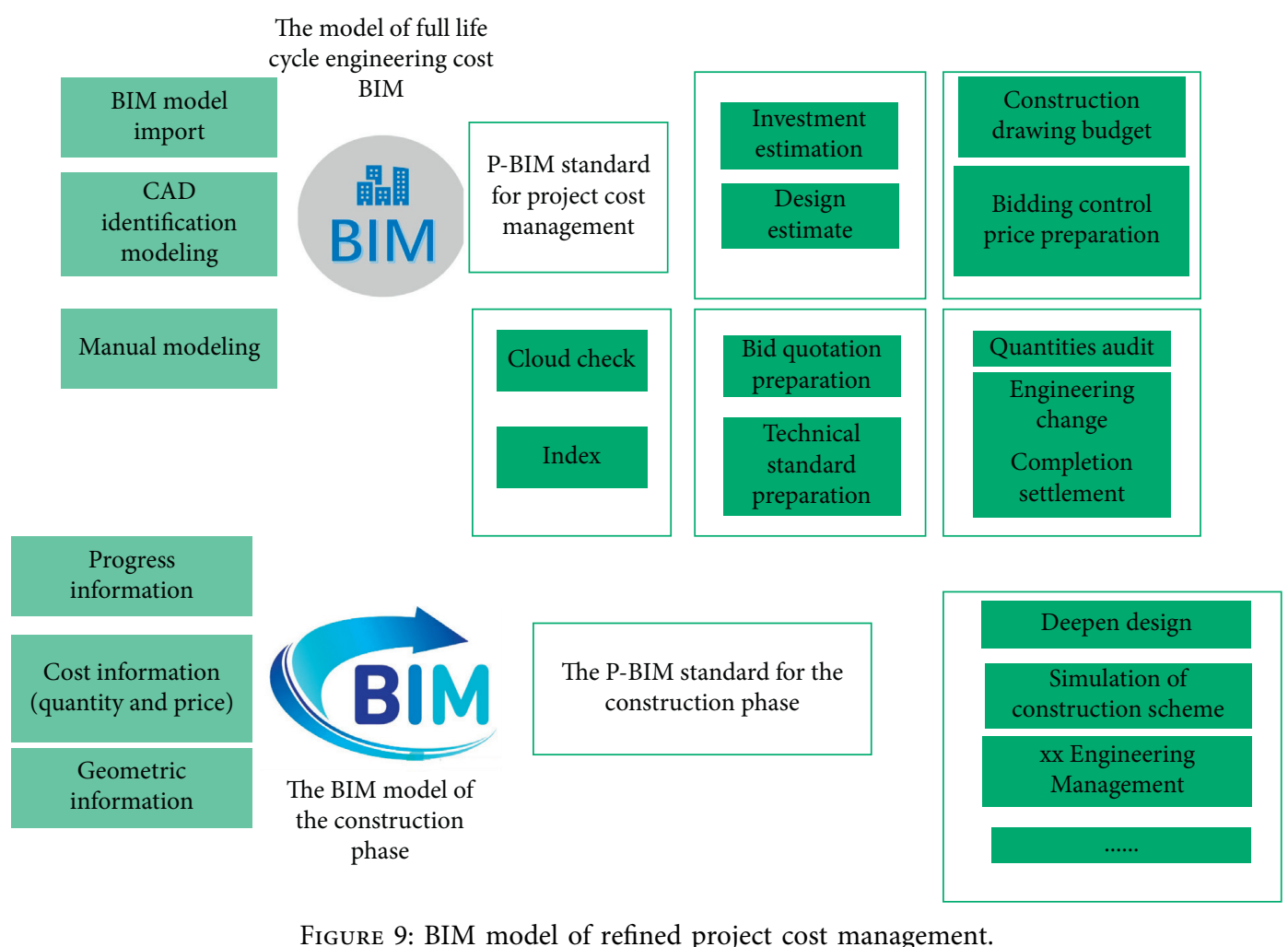

TABLE 1: BIM simulation effect and project cost evaluation effect based on artificial intelligence.

\begin{tabular}{|c|c|c|c|c|c|c|c|c|}
\hline Number & $\begin{array}{c}\text { BIM } \\
\text { simulation }\end{array}$ & $\begin{array}{c}\text { Project cost } \\
\text { analysis }\end{array}$ & Number & $\begin{array}{c}\text { BIM } \\
\text { simulation }\end{array}$ & $\begin{array}{c}\text { Project cost } \\
\text { analysis }\end{array}$ & Number & $\begin{array}{c}\text { BIM } \\
\text { simulation }\end{array}$ & $\begin{array}{c}\text { Project cost } \\
\text { analysis }\end{array}$ \\
\hline 1 & 91.37 & 71.49 & 17 & 91.94 & 78.05 & 33 & 87.85 & 85.47 \\
\hline 2 & 82.68 & 73.21 & 18 & 79.38 & 87.03 & 34 & 91.75 & 81.86 \\
\hline 3 & 87.15 & 74.28 & 19 & 88.21 & 71.28 & 35 & 90.46 & 74.83 \\
\hline 4 & 88.24 & 83.75 & 20 & 87.74 & 85.04 & 36 & 84.11 & 87.69 \\
\hline 5 & 79.13 & 81.51 & 21 & 89.14 & 82.34 & 37 & 80.59 & 84.54 \\
\hline 6 & 79.16 & 88.83 & 22 & 90.57 & 76.98 & 38 & 79.84 & 72.03 \\
\hline 7 & 84.18 & 76.81 & 23 & 87.12 & 87.30 & 39 & 84.89 & 84.56 \\
\hline 8 & 83.85 & 84.15 & 24 & 85.93 & 80.12 & 40 & 91.47 & 78.92 \\
\hline 9 & 86.59 & 87.70 & 25 & 89.30 & 71.47 & 41 & 84.62 & 80.38 \\
\hline 10 & 84.80 & 76.43 & 26 & 86.04 & 71.08 & 42 & 87.68 & 80.46 \\
\hline 11 & 79.47 & 86.08 & 27 & 80.65 & 80.51 & 43 & 79.92 & 82.87 \\
\hline 12 & 91.59 & 83.86 & 28 & 90.69 & 84.05 & 44 & 86.32 & 82.91 \\
\hline 13 & 83.85 & 72.95 & 29 & 79.76 & 84.64 & 45 & 86.80 & 82.51 \\
\hline 14 & 81.48 & 74.85 & 30 & 81.40 & 74.37 & 46 & 84.10 & 84.98 \\
\hline 15 & 79.41 & 80.51 & 31 & 81.72 & 87.15 & 47 & 82.93 & 84.37 \\
\hline 16 & 88.65 & 73.51 & 32 & 82.40 & 76.33 & 48 & 88.72 & 88.32 \\
\hline
\end{tabular}




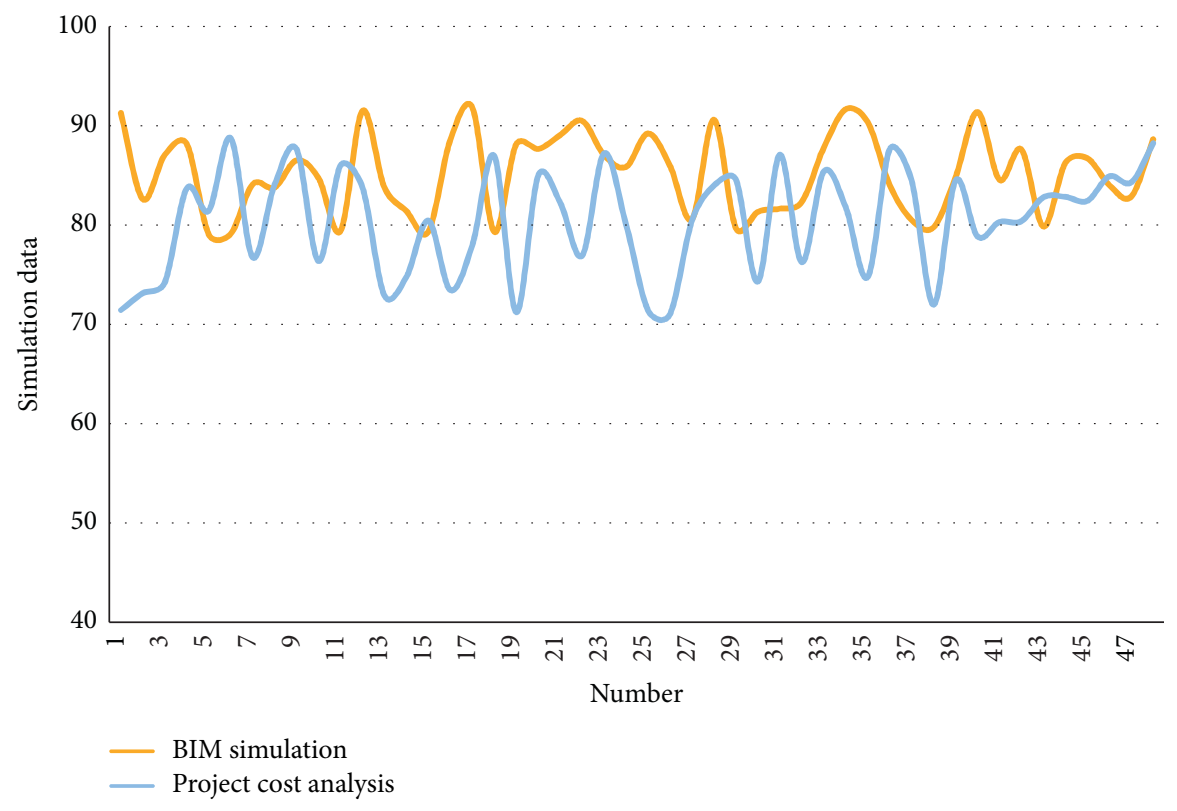

FIGURE 10: Statistical diagram of system performance test data.

Through the above experimental research, we can see that the method proposed in this article has good results.

\section{Conclusion}

In a construction project, different professions such as architecture, structure, materials, and installation will be involved, and it will go through several different stages from decision-making to completion. In this process, different parties of the same information in different professions or at different stages will have different expression and management methods, resulting in information redundancy and difficulties in sharing. The core of BIM is information. The basic element of the BIM model is a single component or object, and the physical characteristics, geometric information, cost information, and construction requirements of all components or objects are expressed through parameters. It then uses 3D Boolean operations and spatial topological relationships to organize these information and store them in a database to finally form a digital model. In addition, the project system based on BIM can adjust relevant information at any time according to project changes, which not only solves the problem of information redundancy but also provides convenient conditions for information exchange among stakeholders.

\section{Data Availability}

The labeled datasets used to support the findings of this study are available from the corresponding author upon request.

\section{Conflicts of Interest}

The author declares no conflicts of interest.

\section{References}

[1] T. Abuimara, W. O’Brien, B. Gunay, and J. S. Carrizo, “Towards occupant-centric simulation-aided building design: a case study," Building Research \& Information, vol. 47, no. 8, pp. 866-882, 2019.

[2] I. Beausoleil-Morrison, "Learning the fundamentals of building performance simulation through an experiential teaching approach," Journal of Building Performance Simulation, vol. 12, no. 3, pp. 308-325, 2019.

[3] A. D. Black, "Wor(l)d-Building: simulation and metaphor at the mars desert research station," Journal of Linguistic Anthropology, vol. 28, no. 2, pp. 137-155, 2018.

[4] D. Chakraborty and H. Elzarka, "Advanced machine learning techniques for building performance simulation: a comparative analysis," Journal of Building Performance Simulation, vol. 12, no. 2, pp. 193-207, 2019.

[5] T. Dodd, C. Yan, and I. Ivanov, "Simulation-based methods for model building and refinement in cryoelectron microscopy," Journal of Chemical Information and Modeling, vol. 60, no. 5, pp. 2470-2483, 2020.

[6] N. Endo, E. Shimoda, K. Goshome, T. Yamane, T. Nozu, and T. Maeda, "Simulation of design and operation of hydrogen energy utilization system for a zero emission building," International Journal of Hydrogen Energy, vol. 44, no. 14, pp. 7118-7124, 2019.

[7] O. Guerra-Santin and S. Silvester, "Development of Dutch occupancy and heating profiles for building simulation," Building Research \& Information, vol. 45, no. 4, pp. 396-413, 2017.

[8] K. Hanson, L. Hernandez, and J. A. Banaski, "Building simulation exercise capacity in Latin America to manage public health emergencies," Health Security, vol. 16, no. S1, pp. S98-S102, 2018.

[9] C. W. Lee and S.-J. Cho, "The development of converting program from sealed geological model to gmsh, COMSOL for building simulation grid," Journal of the Korean Earth Science Society, vol. 38, no. 1, pp. 80-90, 2017.

[10] C. Miller, D. Thomas, J. Kämpf, and A. Schlueter, "Urban and building multiscale co-simulation: case study 
implementations on two university campuses," Journal of Building Performance Simulation, vol. 11, no. 3, pp. 309-321, 2018.

[11] P. Remmen, M. Lauster, M. Mans, M. Fuchs, T. Osterhage, and D. Müller, "TEASER: an open tool for urban energy modelling of building stocks," Journal of Building Performance Simulation, vol. 11, no. 1, pp. 84-98, 2018.

[12] E. K. Wati and N. Widiansyah, "Design of learning media: modeling \& simulation of building thermal comfort optimization system in building physics course," Jurnal Pendidikan IPA Indonesia, vol. 9, no. 2, pp. 257-266, 2020.

[13] C. Xiong, J. Huang, and X. Lu, "Framework for city-scale building seismic resilience simulation and repair scheduling with labor constraints driven by time-history analysis," Computer-Aided Civil and Infrastructure Engineering, vol. 35, no. 4, pp. 322-341, 2020.

[14] X. Xie and Z. Gou, "Building performance simulation as an early intervention or late verification in architectural design: same performance outcome but different design solutions," Journal of Green Building, vol. 12, no. 1, pp. 45-61, 2017.

[15] A. I. Adilkhodjayev, I. M. Mahamataliev, and S. S. Shaumarov, "Theoretical aspects OF structural and simulation modeling OF the macrostructure OF composite building materials," Journal of Tashkent Institute of Railway Engineers, vol. 14, no. 2, pp. 3-14, 2019.

[16] S. Imam, D. A. Coley, and I. Walker, "The building performance gap: are modellers literate?" Building Services Engineering Research and Technology, vol. 38, no. 3, pp. 351-375, 2017.

[17] J.-S. Pei, B. Carboni, and W. Lacarbonara, "Mem-models as building blocks for simulation and identification of hysteretic systems," Nonlinear Dynamics, vol. 100, no. 2, pp. 973-998, 2020.

[18] A. Brunelli, F. de Silva, A. Piro et al., "Numerical simulation of the seismic response and soil-structure interaction for a monitored masonry school building damaged by the 2016 Central Italy earthquake," Bulletin of Earthquake Engineering, vol. 19, no. 2, pp. 1181-1211, 2021.

[19] P. Andrio, A. Hospital, J. Conejero et al., "BioExcel Building Blocks, a software library for interoperable biomolecular simulation workflows," Scientific Data, vol. 6, no. 1, pp. 169-178, 2019.

[20] G. Petrou, A. Mavrogianni, P. Symonds et al., "Can the choice of building performance simulation tool significantly alter the level of predicted indoor overheating risk in London flats?" Building Services Engineering Research and Technology, vol. 40, no. 1, pp. 30-46, 2019.

[21] S. Gibeaux, C. Thomachot-Schneider, S. Eyssautier-Chuine, B. Marin, and P. Vazquez, "Simulation of acid weathering on natural and artificial building stones according to the current atmospheric SO 2/NO x rate," Environmental Earth Sciences, vol. 77, no. 9, pp. 1-19, 2018. 